\title{
Mechanical behavior of the reinforced concrete frame with masonry filling Comportement mécanique des portiques en béton armé avec remplissage en maçonnerie
}

\author{
Jalal KETTAR ${ }^{1}$, Khadija BABA ${ }^{1}$, Abderrahman NOUNAH ${ }^{1}$ and, Lahcen BAHI ${ }^{2}$ \\ ${ }^{1}$ GCE Laboratory, High School of Technology -Sale-, Mohammed V University in Rabat, Morocco \\ ${ }^{2} 3$ GIE Laboratory, Mohammedia School of Engineering, Mohammed V University in Rabat, Morocco
}

\begin{abstract}
Masonry is often used in the most of reinforced concrete structure constructions as a filling material that is important on structural characteristics. Structural contribution in the calculations was neglected or misunderstood, mainly due to the lack of a practical calculation methods and an appropriate regulatory tool.

The analysis of frames filled with masonry is very complex. This complexity is linked from one part to the difference in the nature of elements and its behavior that make up the masonry itself (brick and mortar) and their interaction, and on the other part, for the large dispersion that characterizes the bricks as well as the execution's quality parameters which make it difficult to define reliable criteria for the masonry. The objective of this work is to experimentally highlight the influence of the hollow brick masonry filler, commonly used in Morocco, on reinforced concrete frames subject to lateral stresses, to deepen understanding the seismic behavior of the masonry structures by evaluating the structural performance of a specimen wall. These experimental results will be compared to those found by modeling prototypes, using SAP 2000 software, based on various approaches and models as well as other results deduced from the other researchers. The experimental study was carried out according to standard NF EN 1052-3 on two reinforced concrete frames, of dimensions ( $2 \mathrm{~m} \mathrm{X} \mathrm{1.6m),} \mathrm{the} \mathrm{one} \mathrm{with}$ the masonry filling, and the other without filling in order to determine the initial characteristic resistance to the shearing of the masonry walls. The obtained results showed that a filling has a beneficial effect on rigidity which can be doubled compared to an empty frame. in the same way the lateral resistance. But this effect is much contrasted; it depends a lot on the characteristics essentially of the materials (bricks and concrete). This is the main reason, which justifies the divergence of the results deduced from the nine models that we used.
\end{abstract}

Résumé. Dans la majorité des constructions type structure en béton armé, la maçonnerie est souvent utilisée comme matériau de remplissage dont l'influence sur les caractéristiques des structures est importante. Dans les calculs, sa contribution structurale a été négligée ou méconnue et ce, principalement, en raison de l'absence d'une méthode de calcul pratique ainsi que d'un outil réglementaire approprié. L'analyse des ossatures remplies de maçonnerie est très complexe. Cette complexité est liée d'une part à la différence de nature et de comportement des éléments qui composent la maçonnerie elle-même et à leur interaction et d'autre part, à la grande dispersion qui caractérise les briques ainsi que la qualité d'exécution qui rendent difficile la définition de critères fiables pour la maçonnerie. L'objectif de ce travail est de mettre en évidence expérimentalement l'influence du remplissage en maçonnerie de briques creuses, couramment utilisées au Maroc, sur des portiques en béton armé soumis à des sollicitations de type latéral, en vue d'approfondir la compréhension du comportement sismique des structures en maçonnerie, et ce par l'évaluation de la performance structurelle d'un spécimen de mur. Ces résultats expérimentaux vont être confrontés à ceux trouvés par modélisation de ces prototypes, en utilisant logiciel SAP 2000, s'appuyant sur diverses approches et modèles, et aussi d'autres déduites à partir des résultats d'autres chercheurs. L'étude expérimentale a été effectuée selon la norme NF EN 1052-3 sur deux cadres en béton armé de dimensions ( $2 \mathrm{~m} \times 1.6 \mathrm{~m})$, l'un, avec un remplissage en maçonnerie, et l'autre sans remplissage afin de déterminer la résistance caractéristique initiale au cisaillement des murs en maçonnerie. Les résultats obtenus, montrent qu'un remplissage entraine un effet bénéfique sur la rigidité, qui peut être doublé par rapport à un portique vide. Ainsi que la résistance latérale. Mais cet effet est très contrasté, il dépend beaucoup des caractéristiques essentiellement des matériaux (briques et béton). C'est la raison principale, qui justifie la divergence des résultats déduits à partir des neufs modèles qu'on a utilisé.

Keyswords: Reinforced concrete frame, Walls, Masonry, Hollow bricks, Tests, shearing, Rigidity, Modeling 


\section{Introduction}

La maitrise du comportement des constructions en maçonnerie lors d'un séisme est devenue une nécessité; surtout après les pertes humains et les dégâts matériels très importants, provoqués par le séisme d'AL Hoceima au Maroc le 24 Février 2004, dégâts qui étaient d'autant plus amplifiés que la plupart de ces constructions étaient de type remplissage par maçonnerie. La recherche de méthodes pratiques de calcul, tenant compte du rôle des panneaux de remplissage dans la réponse sismique des structures, passe par la modélisation de la structure sollicitée à échelle 1 , ce qui va permettre de diminuer les imprécisions de calcul. Cette modélisation ne peut se faire sans une bonne compréhension et une évaluation des effets, et principalement l'effort tranchant, que peut causer l'existence du remplissage en maçonnerie sur les structures sollicitées aux séismes et aussi la maitrise des caractéristiques des matériaux constituants les cadres remplis par la maçonnerie.

Cette étude propose une étude expérimentale présentant l'influence des murs de remplissage en maçonnerie sur le comportement des structures en béton armé soumises à un effort latéral. Les résultats expérimentaux seront confrontés à ceux obtenus par modélisations numériques à l'aide du logiciel SAP2000 et basées sur la méthode des bielles. Ce travail vise à améliorer la réglementation en vigueur, à savoir RPS 2011.

\section{Matériels et méthodes}

Nous avons analysé le comportement de murs en maçonneries sous une pression latérale et en quasistatique. Ces essais doivent permettre d'évaluer la capacité portante et le mécanisme de rupture d'un mur en maçonnerie. Dans le montage expérimental, les matériaux de construction les plus courants et qui respectent les standards de la construction au Maroc, ont été utilisés. Cependant, nous avons testé deux spécimens de corps d'épreuve : Le premier, indexé ND1, représente un cadre vide, chargé unidirectionnellement, qui servira de référence. Le deuxième corps d'épreuve, réferencé ND2, représente un cadre identique au premier, mais rempli de maçonnerie de briques creuses (figure 1).

Les deux corps d'épreuve sont soumis à une charge verticale répartie de $6 \mathrm{t} / \mathrm{m}$, constante durant tout l'essai, simulant le poids des étages supérieures. Ils sont en outre soumis à une charge latérale croissante

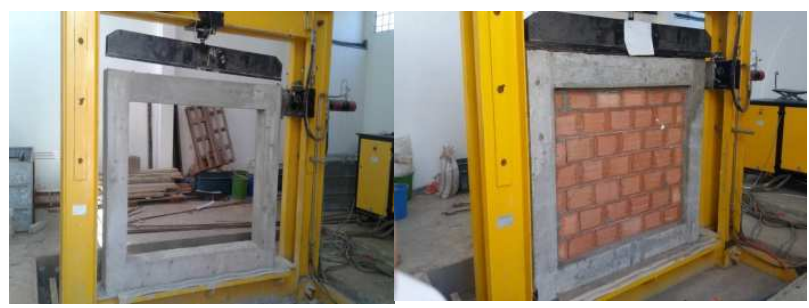

Fig.1a. Cadre vide (ND1) Fig.1b. Cadre rempli (ND2)

Fig.1. Corps d'épreuve

\subsection{Description des corps d'épreuve}

Les dimensions du remplissage sont de $160 \mathrm{~cm}$ pour la largeur (L) et de $120 \mathrm{~cm}$ pour la hauteur (h), soit un élancement de 0,75 .

Le cadre est dimensionné et ferraillé de la même façon, que s'il appartenait au rez-de-chaussée d'un bâtiment courant (le béton est de classe 25/30 et l'acier est de type FeE50). Les poteaux ont une section carrée de 20 × 20 $\mathrm{cm}$, comportant un ferraillage de $4 \mathrm{~T} 12$ et les poutres ont la même section de $20 \times 20 \mathrm{~cm}$ avec un ferraillage constitué de 3 barres T12 en bas et 3 barres T8 en haut; et un ferraillage transversal consitué de cadres T6.

Les panneaux de remplissage sont constitués par une maçonnerie de briques creuses en terre cuite (6 trous), $28 \times 15 \times 7 \mathrm{~cm}$, assemblées par un mortier standard de ciment d'une épaisseur moyenne de $15 \mathrm{~mm}$.

\subsection{Instrumentation et Mesures}

Les écrasements des panneaux sont réalisés par la machine de cisaillement des murs (Marque $3 \mathrm{R}^{\circ}$ ), suivant la Norme NF EN 1052-3. Cette machine est équipée de deux vérins de force identique $(200 \mathrm{KN})$, ces derniers, travaillent avec un système hydraulique, et sont pilotés par un pupitre Quantium, équipés d'un capteur de déplacement (figure 2).

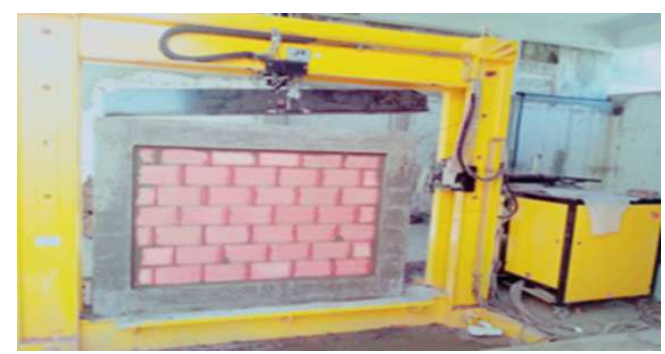

Fig.2. Appareil de cisaillement

\subsection{Modelisation des murs en maçonnerie}

La modélisation des murs en maçonnerie sous chargement latéral se fait selon deux approches soit locale, et bien globale. l'approche locale consiste en la modélisation par éléments finis très complexe censés, représenter la forme réelle des blocs de brique et de mortier, en se basant sur un modèle de l'endommagement, de plasticité, de fissuration ou bien mixte. La complexité de la modélisation rend cette approche très demandeuse en temps de calcul et nécessite une précision au niveau des caractéristiques des matériaux et leurs mise en œuvre. (figure 3). Quant à l'approche globale elle consiste à remplacer le mur en maçonnerie par des éléments simples type barres, soumis à un effort de compression sous forme de bielle, suivant le sens de chargement. (Figure 4).

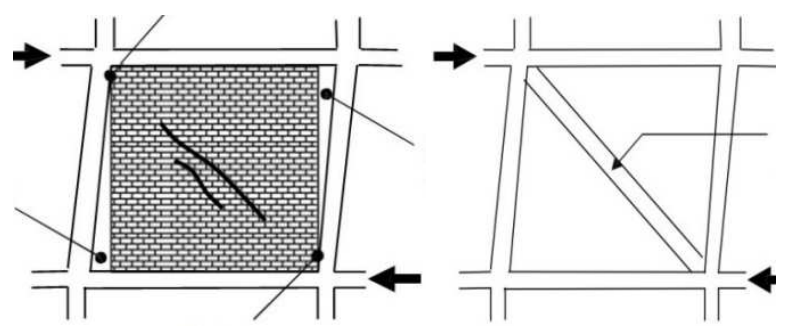

Fig.3. Modélisation par 
Les caractéristiques mécaniques des éléments barres, à savoir le module de Young, le coefficient de Poisson, la résistance à la compression correspondent à ceux de la maçonnerie point sur lequel s'accordent tous les experts, par contre en ce qui concerne les caractéristiques géométriques, et principalement la largeur, de ces bielles, les avis divergent. En effet, plusieurs modèles, basés sur des études expérimentales, ont été établies (Stafford-Smith, 1963[1], Mainstone, 1971[2], klingner et Bertero, 1976[3]).

\subsubsection{Modèle de Mainstone [2]}

Cette approche est la plus utilisée, Mainstone a réalisé plusieurs essais en compression diagonale sur les modèles réduits de cadres et à grande échelle ; utilisant le concept de la bielle diagonale ainsi que le paramètre de la rigidité relative pour étudier la rigidité et la résistance de remplissage. La largeur de la diagonale équivalente $a$ est en fonction de la raideur flexionnelle relative du remplissage et des poteaux constituant le cadre $\lambda$

$$
\lambda=\sqrt[4]{\frac{E_{m} t \sin 2 \theta}{4 E_{c} I_{C} \square}}
$$

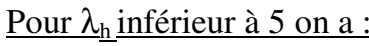

* pour remplissage en brique

$$
\mathrm{a}=0,175 \cdot \lambda^{-0,4} \cdot \sqrt{H^{2}+L^{2}}
$$

* Pour remplissage en béton

$$
\mathrm{a}=0,115 \cdot \lambda^{-0,4} \cdot \sqrt{H^{2}+L^{2}}
$$

Pour $\lambda_{\mathrm{h}}$ supérieur à 5 on a :

* pour remplissage en brique

$$
\mathrm{a}=0,16 \cdot \lambda^{-0,3} \cdot \sqrt{H^{2}+L^{2}}
$$

* Pour remplissage en béton

$$
\mathrm{a}=0,11 \cdot \lambda^{-0,3} \cdot \sqrt{H^{2}+L^{2}}
$$

Avec :

Ec : module d'Young du portique, Ic : inertie de la section du portique,

Em : module d'Young du remplissage,

$\mathrm{t}$ : épaisseur du remplissage,

$\mathrm{L}$ : longueur du remplissage,

$\mathrm{h} ; \mathrm{D} ; \mathrm{H}$ : sont définis sur la figure 5

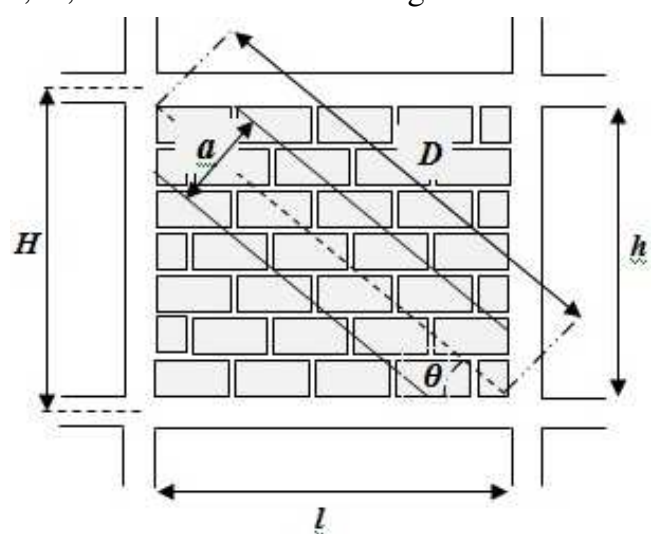

Fig.5.paramètres caractérisant la bielle comprimée équivalente.

\subsubsection{Modèle Elouali [4]}

Il a appliqué les formules de Mainstone [2] sur des structures dont le paramètre de rigidité est inférieur à 5, en corrigeant le coefficient pour l'adapter à ces types de remplissage.

*.Pour les briques d'argiles et de béton :

$$
\text { - } \quad \mathrm{a} / \mathrm{D}=0.135(\lambda \mathrm{H})^{-0.4}
$$

*Pour les briques de chaux et sable :

$$
\text { - } \quad \mathrm{a} / \mathrm{D}=0.175(\lambda \mathrm{H})^{-0.4}
$$

\subsubsection{Modèle de Dawe (Dawe et Al, 1989) [5]}

- $a=\frac{\pi}{1.5 x \lambda_{h}} \cos \theta+\frac{\pi}{1.5 x \lambda_{t}} \sin \theta$

$$
\text { Avec : } \quad \begin{aligned}
\lambda_{t} & =\left(\frac{E_{m} t_{i} \sin (2 \theta)}{4 E_{c} I_{b} l_{i}}\right)^{1 / 4} \\
& \lambda_{\square}=\left(\frac{E_{m} t_{i} \sin (2 \theta)}{4 E_{c} I_{c} \square_{i}}\right)^{1 / 4}
\end{aligned}
$$

$\mathrm{I}_{\mathrm{b}}$ et Ic sont les inerties respectivement des poutres et des poteaux.

\subsubsection{Modèle de Durrani (Durrani \& Al) [6]}

$$
\begin{gathered}
\mathrm{a}=\gamma \sin (2 \theta) D \\
\gamma=0.32 \sqrt{\sin (2 \theta)}\left(\frac{h^{4} E_{m} t_{i}}{m E_{c} I_{c} h_{i}}\right)^{-0.1} \\
m=\left(1+\frac{6 E_{h} I_{h} h}{\pi \varepsilon_{c} I_{c} L}\right)
\end{gathered}
$$

\subsubsection{Modèle de Hrennikoff (Hrennikoff, 1941) [7]}

Le modèle de Hrennikoff est un modèle adéquat pour la modélisation d'un milieu homogène, isotrope, élastique linéaire (Hrennikoff, 1941). Ce modèle a été appliqué pour le béton armé (Kotronis, 2000, Mazars et Al., 2002, Kotronis et al, 2003[8,9 et 10]), nous proposons d'étudier la possibilité d'étendre cette approche à la maçonnerie.

$$
\begin{gathered}
A_{v}=\frac{3}{8} \frac{3 k^{2}-1}{k} a t \\
A_{\square}=\frac{3}{8}\left(3-k^{2}\right) a t \\
A_{d}=\frac{3}{16} \frac{\left(1-k^{2}\right)^{3} / 2}{k} a t
\end{gathered}
$$

Avec t l'épaisseur du voile.

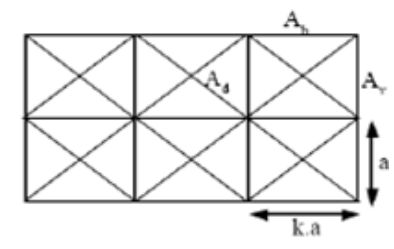

Fig.6.Motif proposé par Hrennikoff

\subsubsection{Modèle de Liauw et Kwan 1984 [11]}

Il a travaillé sur l'analyse des portiques remplis par une approche par éléments finis incrémentale et itérative, qui compte des non-linéarités des matériaux et du 
comportement structural de l'interface cadre-remplissage. La méthode utilise une procédure itérative à déplacement incrémental basée sur la rigidité sécante.

La largeur de la diagonale est :

$$
a=\frac{0,95 \cdot H \cdot \cos \theta}{\sqrt{\lambda_{\square}}}
$$

\subsubsection{Modèle de Priestly 1992 [12]}

Il a supposé que la largeur de la diagonale est constante sur toute sa largeur sans prendre en compte les propriétés de cadre de remplissage. La largeur de la diagonale est donnée par la formule suivante :

$$
\mathrm{a}=0.25 d
$$

Avec d : longueur de la diagonale de remplissage.

\subsubsection{Modèle de Cruz-Diaz 2002 [13]}

La méthode utilisée par Cruz-Diaz met en évidence la corrélation entre la largeur équivalente de la diagonale et la longueur des briques de maçonnerie.

$$
a=l\left(\frac{l}{l_{c}}\right)^{r}
$$

Avec 1 : longueur des briques

$\mathrm{r}, \mathrm{lc}$ : paramètres de calage, on peut prendre pour :

*. Joints verticaux remplis : $\mathrm{r}=0.545 ; \mathrm{lc}=2.247$

*. Joints verticaux secs : $r=0.876 ; 1 \mathrm{c}=0.384$

\subsubsection{Modèle adopté par le règlement PS92[14]}

Le principe de calcul de résistance consiste à assimiler l'ensemble formé par les panneaux de maçonnerie et par les chaînages en béton armé qui les encadrent à un système triangulé dont les éléments diagonaux sont constitués par les bielles actives susceptibles de se former dans la maçonnerie. La largeur a de ces bielles est prise, dans les calculs, égale à la plus petite des deux valeurs $\mathrm{d} / 6$ et $4 \mathrm{e}$, soit :

$$
\mathrm{a}=\min (\mathrm{d} / 6 ; 4 \mathrm{e})
$$

Avec : d est la longueur de la bielle (diagonale du panneau), e est l'épaisseur brute de la maçonnerie

\subsubsection{Modèle de FEMA [15]}

$$
a=0.201 \frac{\sqrt{H^{\prime 2}+L^{\prime 2}}}{H^{0.4}\left(E_{d} \cdot S \cdot \sin (2 \theta)\right)^{0.1}}\left(E_{f} I_{c} H^{\prime}\right)^{0.1}
$$

$$
\text { Avec } \theta=\tan ^{-1}\left(\frac{H^{\prime}}{L^{\prime}}\right)
$$

Avec $\mathrm{s}$ et $\mathrm{E}_{\mathrm{d}}$ : la largeur et le module de rigidité $\mathrm{du}$ remplissage, $\mathrm{E}_{\mathrm{f}}$ : le module de Young du Portique $\mathrm{H}$ et $\mathrm{L}$ sont la hauteur et la longueur du portique, H'et L' la hauteur et la longueur du remplissage, I : moment d'inertie de la section du poteau

\section{Résultats et discussions}

\subsection{Largeur de la bielle du cadre ND2}

Lors de suivi du chargement horizontal du corps d'épreuve ND2 nous avons noté que le cadre se séparait du remplissage au niveau de la diagonale tendue, avec une largeur mesurée de l'ordre de $37 \mathrm{~cm}$, (figure 8), valeur qui est en concordance avec la théorie de la diagonale comprimée équivalente de largeur a et qui varie, pour des panneaux carrés, entre 0.3 à 0.4 (Stafford Smith 1963[1])

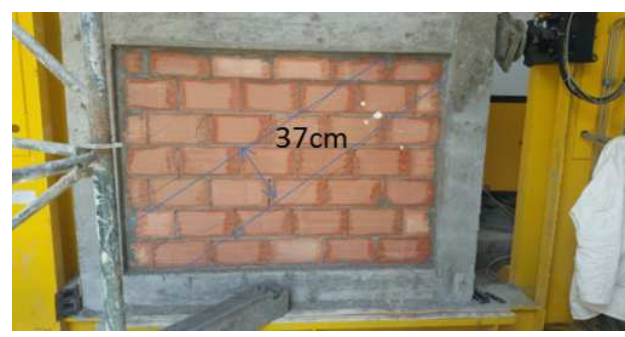

Fig.8: Diagonale comprimée équivalente

L'histogramme ci-dessous (figure 9), synthétise les valeurs obtenues à partir des modèles énumérés ci-dessus. Nous pouvons dire que le modèle de Durrani [6] coïncide presque parfaitement avec les résultats expérimentaux avec une erreur de 5\%, pour le modèle adopté par le règlement parasismique Français PS 92 [12], donne des résultats relativement éloignés avec une incertitude de $24 \%$, par contre les autres modèles sont un peu loin avec des erreurs variant de $35 \%$ à $75 \%$, sauf pour le modèle de Dawe 1989[5], donne des erreurs excessives de l'ordre de $200 \%$, (cette valeur de Dawe[5] est en concordance avec les résultats obtenus par Samouh [16]). Ceci est dû principalement à la diversité des corps d'épreuves utilisés. Et les matériaux utilisés.

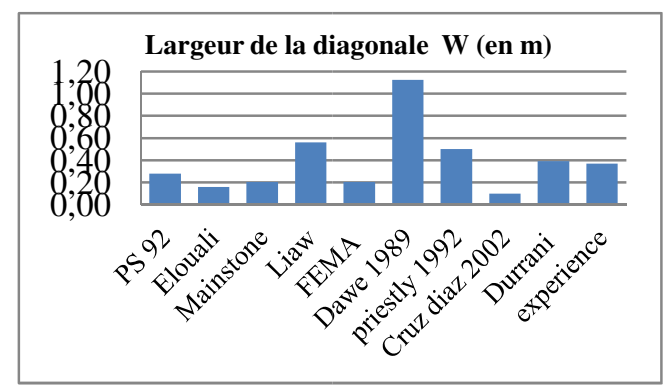

Fig.9: Largeur de la bielle diagonale a (en m)

\subsection{Etude comparative du comportement des corps d'épreuve (ND1 \& ND2)}

Lors de l'interprétation de ces deux diagrammes (figure 10), qui représentent la loi de comportement de mur rempli (ND2) et celui du mur vide (ND1), on peut conclure que le remplissage améliore nettement la rigidité, ainsi que la résistance à la rupture

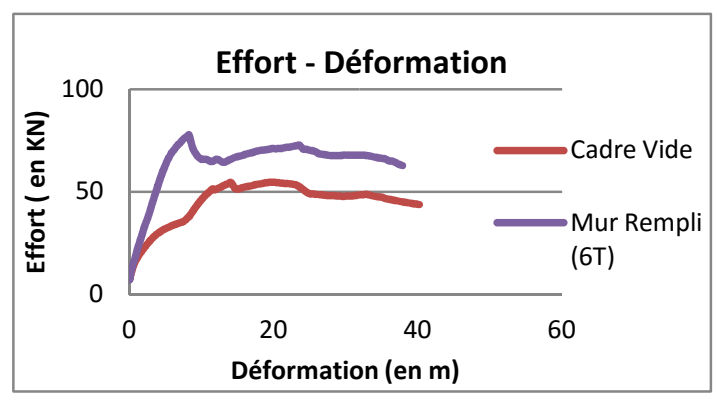

Fig.10 : Loi de comportement (Effort- Déformation)

En effet : La résistance à la rupture, s'est améliorée de presque $42 \%$, grâce à l'ajout du remplissage (de 54 à 77 $\mathrm{KN}$ ). Ainsi que de la même manière, la première fissuration s'est retardée avec la présence du remplissage, 
ce qui agit sur le déplacement limite élastique qui s'est apprécié presque de $80 \%$, par conséquent, la rigidité du portique s'est doublée, pour leND2. Ces résultats sont conformes à ceux obtenus par d'autres chercheurs, en particulier Irwin (1979) dont les valeurs étaient comprises entre 2,7 et 8,65 .

Cette conclusion peut être justifiée analytiquement par les formules théoriques prononcées par le règlement Marocain Parasismique RPS 2011[17].

La rigidité totale est composée selon le RPS 2011 de deux termes, à savoir La rigidité apportée par le cadre $(\mathrm{Kc})$ et celle apportée par le remplissage $(\mathrm{Kr})$, ces derniers sont déduites à partir des deux formules suivantes :

$$
\mathrm{K}=\mathrm{Kp}+\mathrm{Kr}
$$

Avec Kp : Rigidité latérale du portique

$$
\begin{aligned}
& \mathrm{Kp}=12 \sum(\text { Ec.Ic }) / \mathrm{h}^{3}(\mathrm{~L}+2 . \lambda) \\
& \lambda=\mathrm{Ls} . \mathrm{Ic} / \mathrm{hs} . \mathrm{I}
\end{aligned}
$$

$\sum$ sur le nombre des travées.

$\mathrm{Kr}$ : La rigidité latérale du panneau :

Kr $=\mathbf{0 . 0 4 5} \mathbf{. m}$. $\left(\right.$ Er.e $\left.\cos ^{2} \boldsymbol{\alpha}\right)$
$\mathrm{m}:$ Nombre des travées
Er : Module d'élasticité
.e : épaisseur du panneau
$\alpha:$ est l'angle de la diagonale avec
l'horizontale du panneau
Les résultats trouvés par les différents modèles précités ci-dessous en appliquant ces dernières formules de la rigidité, sont résumés dans l'histogramme ci-dessous, (figure 11);

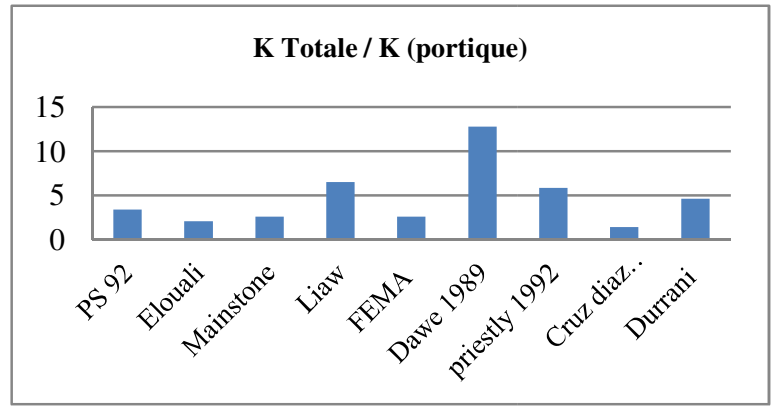

Fig.11 : Rapport des rigidités (Ktotale /Kportique)

Les modèles de Mainstone[2], d'Elouali[4] et FEMA[15], confirme ce résultat intéressant, que le rapport des rigidités est de l'ordre de 2. Cruz diaz [13], a obtenu 1.4 ; par contre les autres ont trouvé des valeurs comprises entre 3 et 6 , sauf bien sûr, le modèle de Dawe[5], qui diverge complètement comme pour la largeur de a bielle. Ceci peut s'expliqué par le calibrage des modèles, en effet: Le modèle d'Elouali[4] s'est basé sur celui de Mainstone[2], et de la même manière FEMA[15], et en plus La formule de calcul sur laquelle on s'est basée est déduite du RPS 2011, édité par T Elouali.[4] Mais ce qui est sure, que le remplissage augmente d'une manière significative

\section{3 Étude comparative entre un mur vide modélisé et après expérience}

Sur le tableau ${ }^{\circ} 1$, nous avons reporté les valeurs obtenus suite à la modélisation numérique par le logiciel éléments finis SAP2000, On peut valider notre modèle car l'erreur trouvée par l'expérience, pour le déplacement, est de presque $3 \%$, par contre on a trouvé une erreur de l'ordre $9.5 \%$ concernant la rigidité

Tab.1:Résultats expérimentaux vs par modélisation

\begin{tabular}{|l|l|l|}
\hline & $\begin{array}{l}\text { la rigidité } \mathrm{K} \\
(\mathrm{MN} / \mathrm{m})\end{array}$ & $\begin{array}{l}\text { Le déplacement } \\
(\mathrm{mm})\end{array}$ \\
\hline $\begin{array}{l}\text { Résultats } \\
\text { expérimentaux }\end{array}$ & 4.01 & 11.22 \\
\hline $\begin{array}{l}\text { Valeurs SAP } \\
2000\end{array}$ & 4.39 & 10.86 \\
\hline
\end{tabular}

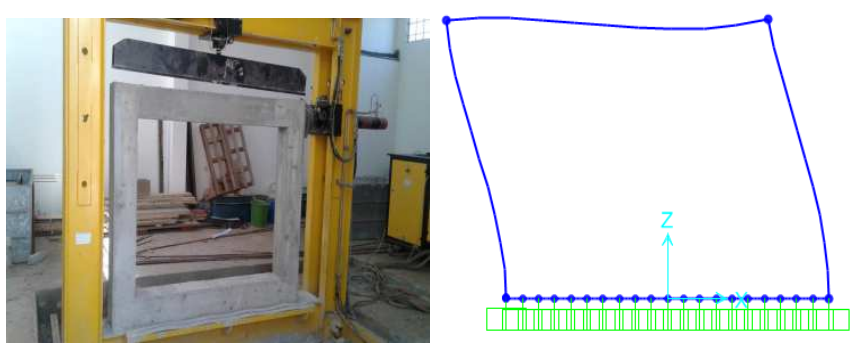

Fig.12 : Modèle du Cadre vide
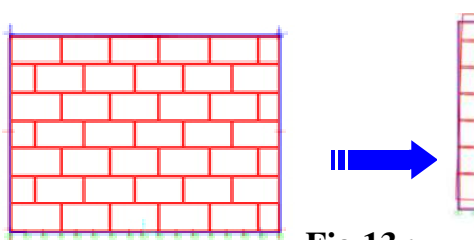

Fig.13 :

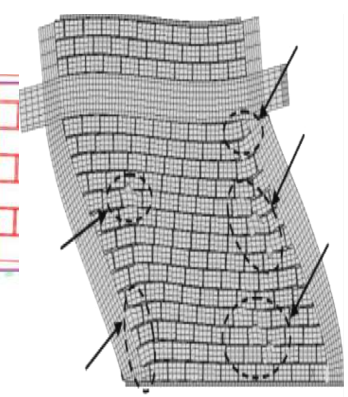

Modèle du Cadre Rempli

On peut noter également que la contrainte limite élastique n'a augmenté que de $7 \%$ alors que la contrainte de rupture à augmenter de $150 \%$, chose que l'on peut justifier par le comportement non linéaire de ce type de structure, et que de ce fait on peut dire que l'effort est repris en premier lieu par le cadre, jusqu'à fissuration et par la suite le chargement est transmis au remplissage en maçonnerie prend la relève pour équilibrer l'effort supplémentaire.

D'après l'essai de cisaillement du mur rempli, nous avons reporté une cartographie de fissuration très proche du modèle homogénéisé, ceci confirme la validité de ce modèle. 


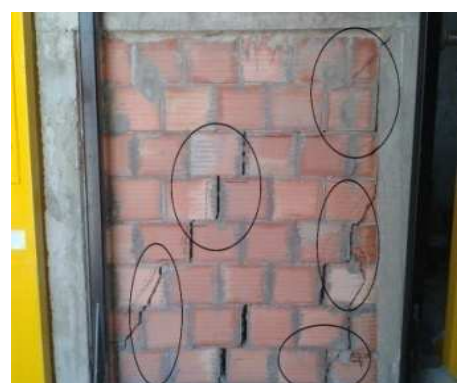

Fig.14 : Mode de rupture du mur rempli

\section{Conclusion}

Au terme de cette étude, et d'après l'exploitation des résultats obtenus, nous pouvons déduire que :

- La méthode des bielles est bien adaptée à ce genre de structures, elle est aussi justifiée par l'expérience.

- La présence du remplissage en maçonnerie peut jouer un rôle bénéfique en réduisant les déplacements horizontaux et les contraintes de flexion.

- l'effort tranchant et le moment fléchissant se trouvent réduits par la présence du remplissage.

- la présence du remplissage change le mode de déformation et de déplacement.

\section{Références}

1. Stafford Smith, B., "Lateral Stiffness of Infilled Frames.”Journal of Structural Division, ASCE, 1962, Vol. 88, No.ST6, pp. 183-199.

2. R. J. Mainstone, [1971], On the stiffnesses and strengths of infilled frames,Proc. of the Institution of Civil Engineers, Supplement (IV), Paper 73605:57-90.

3. R. E. Klingner, V. V. Bertero; Infilled Frames in Earthquake-resistant Construction, University of California, 1976 - 548 pages

4. T. Elouali (1998) Thesis Ph.D. "Effects of infill panels on the seismic response of frame buildings.

5. JL. Dawe, CK. Seah. Behavior of masonry infilled steel frames. Can J Civ Eng 1989;16:865-76.

6. A.J Durrani, Y.H. Luo, Seismic retrofit of flat slab buildings with masonry infill, Nceer Workshop on Seismic response of masonry infills, SanFrancisco, 1994

7. A. Hrennikoff A., «Solution of Problems of Elasticity by the Frame-Work Method ». ASME J. Appl. Mech., $\mathrm{n}^{\circ}$ 8, 1941, p. A619- A715.

8. P. Kotronis, Cisaillement dynamique de murs en béton armé. Modèles simplifiés 2D et 3D. Matériaux. École normale supérieure de Cachan, ENS Cachan, 2000.

9. J. Mazars, L. Davenne, P. Kotronis, F. Ragueneau, Casaux G.A 3D beam element analysis for R/C structural walls'. 12th European Conference on Earthquake Engineering, cd paper no 751, London 9-13 September 2002

10. P, Kotronis, J. Mazars, L. Davenne, 'The equivalent reinforced concrete model for simulating the behavior
- Le remplissage entraine un effet bénéfique sur la rigidité, qui peut être doublée par rapport à un portique vide.

- De la même manière la résistance latérale. Mais cet effet est très contrasté, il dépend beaucoup des caractéristiques essentiellement des matériaux (briques et béton). C'est la raison principale, qui justifie la divergence des résultats déduits à partir des neufs modèles qu'on a utilisé.

- L'utilisation des modèles déjà connus pour dimensionner des structures, en particulier PS 92, à utiliser avec méfiance, car ces derniers sont calés sur des matériaux, et des techniques de mise en œuvre, différents par rapport qu'on utilise chez nous au Maroc. D'où la nécessité d'approfondir cette recherche, en multipliant les essais, afin d'élaborer un nouveau modèle tenant compte de ces contraintes

- A partir des diagrammes des lois de comportement (contraintes-déformation), on peut conclure que le mur rempli a un comportement non linéaire, et par conséquent, toute modélisation doit tenir compte de ce comportement; nous recommandons l'utilisation de la méthode non linéaire. 
of shear walls under dynamic loading '. Engineering Fracture Mechanics, vol. 70, issues 7-8, pp. 1085-1097, 2003.

11.. T. C. Liauw and K. H. Kwan, "Nonlinear Behavior Of Non-Integral Infilled Frames," Computers \& Structures, vol. 18, pp. 551-560, 1984.

12. T. Paulay and M. Priestley, Seismic Design of Reinforced Concrete and Masonry Buildings. New York: Jhon Wiley \& Sons, 1992.

13. J.I.Cruz Diaz, Etude des murs de contreventement en maçonnerie d'éléments de terre cuite Thèse l'Université de Marne-la-Vallée (2002)

14. DTU NF P 06-013. Règles de construction parasismique - Règles PS applicables aux bâtiments, dites règles $\mathrm{PS} 92$

15. «FEMA 273, 306 et 356» Federal Emergency Management Agency, USA, 1997,1998 et 2000

16. H. Samouh et All: Modélisation simplifiée des portiques avec remplissage en maçonnerie soumise à l'action sismique :conference paper sept 2011 ; DOI : 10.13140/2.1.4756.1761

17. «Regalement parasismique Marocain»(RPS2011);

18. M. Holmes, Steel frames with brickwork and concrete filling. Proceedings of the Institute of Civil Engineers 1961: 19(6501): 473-478.

19. H. Cherif, Modélisation simplifiée des portiques avec remplissage en maçonnerie soumis à l'action sismique test. Mast-Gc.

20. K, Lefebvre, Etude du comportement sous charges latérales des ossatures de béton armé avec murs de remplissage de maçonnerie, construites avant les années 1960 (2012) ; thèse présentée à l'école de technologie supérieure canada

21. T. Telford, Rc Frames under earthquake loading, state of the art report, Comité Euro-International Du Béton.

22. NF EN 1996 Eurocode 6 : Calcul des ouvrages en maçonnerie

23. NF EN 1052-3 Avril 2003 Méthodes d'essai de la maçonnerie - Partie 3 : détermination de la résistance initiale au cisaillement

24.F. B. HOUTI, Comportement des structures en maçonnerie sous sollicitation uni axiale, Universite Abou Bekr Belkaid - Tlemcen, 2002

25. N. Mezigheche, Comportement des remplissages dans les structures sous différentes sollicitations: cas de la maçonnerie, Mémoire de Magister, Faculté Des Sciences de l'Ingénieur,2010.

26. T. Bouchakour, Interaction entre ossature en béton armé et maçonnerie de briques creuses sous sollicitations de type sismique, Thèse de Doctorat, Mechanics. Ecole Nationale des Ponts et Chaussees, 1984.

27. A. Ghanem, Analyse non linéaire des structures planes en béton armé tenant compte du remplissage en maçonnerie, Mémoire de Magister, Université Mouloud Mammeri De Tizi-Ouzou, 2013.

28. I. Ait Ramdane, Comportement sismique d'ossatures en portiques béton armé en tennant compte de l'interaction murs de remplissage en maçonnerie-cadre des portiques, Mémoire de Magister, Université Mouloud Mammeri De Tizi-Ouzou, Faculté du Génie de la Construction, 2014 